\title{
EVENT-RELATED POTENTIALS EXTRACTION OF WORKING MEMORY USING WAVELET ALGORITHM
}

\author{
SitiZubaidahMohd Tumari, Rubita Sudirman and Abdul Hamid Ahmad \\ Department of Electronics and Computer Engineering, Universiti Teknologi Malaysia, Johor Bahru, Malaysia
}

Received 2013-10-02; Revised 2013-10-17; Accepted 2013-11-13

\begin{abstract}
This study was designed to classify and determine the Event-Related Potentials (ERPs) signal pattern of normal children on visual response. Thirty-eight children aged between 10 to 12 years old were subjected to a two-phase computer-based assessment while their working memory activity was recorded using a NeurofaxEEG 9200 machine. For children, it is anticipated that some information can be lost when there is too much information given at any one time due to limited memory capacity and this is a type of memory impairment. Based on the visual stimulus responses, EEG signal were recorded and captured from channel location at Fz. This paper explains the extraction of raw EEG signals into grand mean ERPs signal which to determine the pattern of signal developed. The ERPs concerning latency and amplitude variability of the P300 component was evaluated. The analysis was based on Discrete Wavelet Transform (DWT) algorithm and focused on alpha rhythm. Results indicated that the Daubechies wavelet at a decomposition level of 4 (db4) was the most suitable wavelet for pre-processing raw EEG signal of working memory. A significant increase of latency was detected in children aged 10 to 12 years old at channel Fz (frontal midline) when the visual stimuli became more difficult. For amplitude variability, the girls gave higher amplitude at Phase 1. These results supported the concept of increased cognitive memory in children.
\end{abstract}

Keywords: Visual Stimuli, ERPs, P300, DWT, Amplitude

\section{INTRODUCTION}

Our working memory is a temporary memory that stores and manipulates information in our brains. Its recent researches include differentiating between what our brains develop and perceive as simple or complex spans. Tasks recognized as complex span are things that are remembered using numerous frameworks of disturbing task, for example, answering mathematics question and interpreting sentences. On the other hand, simple span tasks are about unloading primary and secondary memory such as words and letters (Pritzer and Blankenburg, 2011). In this study, more attention is given to complex span events in children.

Human are able to store about 1 to 10 items for a short period of time in the active state for about 0 to 60 sec, depending on the individual cognitive memory capacity (Potagas et al., 2011). Impairments in working memory may cause an individual to forget verbal instructions, face difficulties in solving simple problems and confuse oneself while multitasking.

The prefrontal cortex area of the brain records the performance of our working memory (Tumari et al., 2012). It is at the anterior part of the brain as shown in Fig. 1 and focuses on problem solving, emotion and complex thought. Associated brain imaging studies have proven that the prefrontal cortex can present a continuous activity through a delay phase of visual stimuli (Donohue et al., 2013).

Nowadays, more and more children are facing short term memory impairments. As such, this study was carried out to identify the pattern of the children's Event-Related Potentials (ERPs) signal while being engaged in two visual stimuli events. The children's responsiveness to visual stimuli concerns their ability to interpret the surrounding environment after processing the information contained in visible light. By using visual stimuli events, the interactive and neuronal consequences of impulsive positioning can be examined (Krauzlis et al., 2013).

Corresponding Author: SitiZubaidahMohd Tumari, Department of Electronics and Computer Engineering,

Universiti Teknologi Malaysia, Johor Bahru, Malaysia 


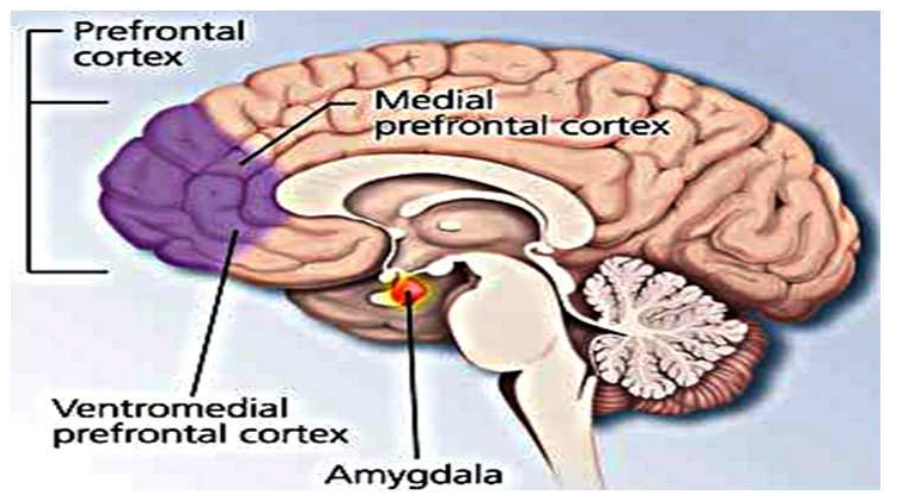

Fig. 1. Medial view of prefrontal cortex

\subsection{Electroencephalography (EEG)}

Electroencephalography (EEG) is used to observe the functional integrity of prefrontal cortex activity in children. It is a kind of medical imaging technique that records the electrical activity generated by brain structures (Sudirman et al., 2010). The electrode at the low impedance is less than $10 \mathrm{k} \Omega$ and is used to provide an accurate detection of the voltage of the brain neuron current. However, impedances that are too low can cause signal distortion. In this study, the acquisition of working memory data was recorded using Neurofax-EEG 9200.

The EEG is generally designated in terms of rhythmic activity, which is separated into frequency bands that can be further categorized into five-alpha, beta, gamma, delta and theta bands (Table 1). The best-known and most extensively studied rhythm of the human brain is the normal alpha rhythm (8 to $14 \mathrm{~Hz}$ ). As the EEG procedure is non-invasive and painless, it is used to investigate the brain organization of cognitive processes such as perception, memory, attention, language and emotion in normal children. The amplitude and frequency of alpha rhythm are known to be affected by external stimuli such as light and sound, memory tasks and mental arithmetic (Nenert et al., 2012).

\subsection{Event-Related Potentials Processing}

Event related potentials are initiated by neuronal mass activity that can generate measurable electric scalp fields in fixed-time relation to an event to find a similar effects of inversion and contrast reversal behaviorally on reflecting various stimuli (Yang et al., 2013). Analyzing the ERPs can serve cognitive purposes in assessing cognitive responses without recognizable performances, but with neurophysiologic activity recorded.

Event-related potentials like N200, P300, N400 and P600 as well as error-related potentials are not the only ones transient temporal graphic-elements used in brain analysis (Saavedra and Bougrain, 2010). ERPs can be generally categorized into two hypotheses that concern specific and non-specific effects. The hypothesis of specific effect predicts how ERPs waveform varies between conditions and is constrained by temporal and spatial information. Non-specific hypothesis focuses on the difference in neuronal response between conditions. Amplitude and latency variability of the P300 component occurs when there is a specific effect. For stimulus complexity, P300 has a more significant behavior for complex span while doing visual stimuli. This study has focused on alpha range because the ERPs are within the low frequency bands.

\subsection{Wavelet Algorithm}

In order to study the behavior of ERPs, the related amplitude and latency were measured at different frequency peaks. The advantage of using wavelet transformation rather than Fourier transformation is that wavelet algorithm can capture the transient features in a given signal and provide the corresponding time frequency information. The Discrete Wavelet Transform (DWT) was chosen to decompose the signal through the means of low-pass filter (cAs) and high-pass filtering (cDs). To perform the required decomposition, the output signals having half the frequency's bandwidth from the original signal have to be down-sampled by 2 using Nyquist rule.

Referring to this rule, an original signal has a highest frequency of $\omega$, this involves a sampling of $2 \omega$ before raw signal can be down-sampled by to $\omega / 2$. The down sampling of a time domain signal can be divided into two which are low and high filtering. Down-sampling occurs when the original signal, $\mathrm{x}(\mathrm{n})$, that passes through a half band high-pass filter, $\mathrm{g}(\mathrm{n})$, detail coefficient and then into a low-pass filter, $h(n)$ (approximation coefficient) (Oliveira and Bretas, 2009). 
Table 1. Brain wave activity and frequency bands (Sanei and Chambers, 2007)

\begin{tabular}{lll}
\hline Activity & Frequency $(\mathrm{Hz})$ & Descriptions \\
\hline Delta & $0-4$ & Deep sleep, lucid dreaming, hypnosis \\
Theta & $4-8$ & Deep relaxation, meditation, focus, creative, hypnagogic state \\
Alpha & $8-14$ & Light relaxation, positive thinking, fast thinking \\
Low beta & $14-15$ & Relaxed focus, improved attentive abilities \\
Mid-beta & $15-18$ & Increase mental ability, focus alertness, IQ \\
High beta & $18-32$ & Fully awake, normal state of alertness, stress and anxiety \\
Gamma & $32-60$ & Associated with information rich task processing and high frequency \\
\hline
\end{tabular}

Table 2. Decomposition of EEG signal into different frequency bands $\left(\mathrm{F}_{\mathrm{s}}=1000 \mathrm{kHz}\right)$

\begin{tabular}{lll}
\hline $\begin{array}{l}\text { Frequency } \\
\text { range }(\mathrm{Hz})\end{array}$ & $\begin{array}{l}\text { Decomposition } \\
\text { level }\end{array}$ & $\begin{array}{l}\text { Frequency } \\
\text { band }\end{array}$ \\
\hline $1000-500$ & D1 & Noise \\
$250-500$ & D2 & Noise \\
$125-250$ & D3 & Noise \\
$63-125$ & D4 & Noise \\
$31-63$ & D5 & Gamma \\
$16-31$ & D6 & Beta \\
$8-16$ & D7 & Alpha \\
$4-8$ & D8 & Theta \\
$0-4$ & A8 & Delta \\
\hline
\end{tabular}

A one level of decomposition can be mathematically expressed as in Equation (1 to 3) (Olkkonen, 2011):

$$
\begin{aligned}
& y(n)=x(n) \times h(n) \\
& =\sum_{k=1}^{N} x(k) \times h(n-k) \\
& =\sum_{k=1}^{N} h(k) \times x(n-k) \\
& y_{\text {high }}(k)=\sum_{n} x(n) \times g(2 k-n) \\
& y_{\text {low }}(k)=\sum_{n} x(n) \times h(2 k-n)
\end{aligned}
$$

Where:

$$
\begin{array}{ll}
\mathrm{x}(\mathrm{n}) & =\text { Original signal } \\
\mathrm{h}(\mathrm{n}) & =\text { Low-pass filter } \\
\mathrm{g}(\mathrm{n}) & =\text { High-pass filter } \\
\mathrm{y}_{\text {high }}(\mathrm{k}) & =\text { Outputs of the high-pass filter } \\
\mathrm{y}_{\text {low }}(\mathrm{k}) & =\text { Outputs of the low-pass filter }
\end{array}
$$

In accordance to the theory presented above, the multiresolution analysis was performed with Daubechies wavelet set as 4 and level decomposition set to 8 since the frequency sampling rate was $1000 \mathrm{kHz}$. In this representation, the coefficients A1, D1, A2, D2, A3, D3, A4, D4, A5, D5, A6, D6, A7, D7, A8 and D8 were the frequency content from the original signal within the bands. The extracted wavelet coefficients provided a compact representation of different frequency ranges, as shown in Table 2. Original signal content the length of frequency is 1000 and from 1000 lengths it will down sampling by 2 . Low frequency contents length from 0 to 500 as name as approximation coefficient (A1) and high frequency (500 to 1000) as a detailed coefficient (D1). Then, at A1 (low frequency) will continue down sampling by 2 which are A2 (0 to 250) and D2 (250 to 500). The processes of down sampling continue until the original signals have found all the frequency ranges.

The lower panel discloses the wavelet decomposition of the average ERPs. The coefficients show the correlation of the average ERPs with the wavelet function at different scales (D1-D8, A8) and times. For the P300 component, the largest positive peak is at about $400 \mathrm{~ms}$. An appearance of this wavelet family is ideal in the acquisition of ERPs due to their compact support, regularity and symmetry (Tumari et al., 2013). In this study, the coefficients of the scale level corresponding to the alpha band were submitted for further analysis. For each subject, the alpha coefficients of the 30 single sweeps were averaged and then compared statistically. Finally, the results for all subjects were averaged to obtain a grand average. Results indicated that the temporal resolution of the scale corresponding to the alpha band was $64 \mathrm{~ms}$.

\section{MATERIALS AND METHODS}

\subsection{Participants}

Data were collected from 38 healthy young volunteers from a primary school or more specifically, 19 boys aged $10-12$ years and 19 girls aged 10-12 years old. They had no previous history of neurological and mental abnormalities records and were selected based on their classroom assessment performance related to remembering certain skills to achieve a representative sample on experiment consequences.

\subsection{Task and Procedure}

In this study, the events involved the participants' reaction to different pictures and their ability to give an appropriate response within a certain reaction time period. The reaction time was measured between the onsets of the stimulus and the reflection on the entire 
processing. To be more precise, the children were given some visual stimuli to investigate the responses of their brain activity while remembering the sequence of the pictures. The children were asked to relax for $30 \mathrm{sec}$ before the assessments started. This assessment was divided into two phases-Phase 1 (the Study Phase) and Phase 2 (the Memory Phase).

\subsubsection{Phase 1: The Study Phase}

At this stage, the participants had to study several pictures presented to them on a computer screen. Figure 2 shows four sample pictures that have been presented to the children. Every picture was displayed for $5 \mathrm{sec}$ and the whole sequence was repeated twice. After that, the children were prompted with a few questions about the pictures and their responses were recorded using the EEG machine.

\subsubsection{Phase 2: The Working Memory Test Phase}

During the working memory test phase, a delayed match-to-sample task was modified by inserting new pictures. Each memory trial consisted of a sample target (new and studied) and several test pictures (matched targets and studied distracters). Figure 3 shows the test pictures that were repeated twice in a given trial. The target sample was presented for $5 \mathrm{sec}$ and the new sample (fixation picture) was presented for a second or more between any two sample targets. All participants had to response as quickly and accurately as possible.

\subsection{EEG Signal Processing}

The signal was recorded using 10-20 Standards Electrode Placement System based on the Event-Related Potentials (ERPs) method. Figure 4 shows the experimental setup to collect the data. EEG recording were obtained by placing wired electrodes on the participants' scalp. The sampling frequency was set to $1000 \mathrm{~Hz}$. The participant sit happily in front of computer monitor in sound proofed room with dim light and look at the viewed seen images. The distance between children and monitor must constant for every participants because want to evade artifacts or noise. Artifacts may also be biological when they originate from the internal side of the body, such as from eye activities (blinking and movements of eye muscle), electromyography activities and kinetic activities (tongue movements). After that, the participants need to observe and remember the efficiency of the pictures within the time stipulated. The raw of data were saved in ASCII code and converted into .txt files in MATLAB software. The signal was decomposed by using Daubechies order $4(\mathrm{db} 4)$ as a mother wavelet for the pre-processing.

Preprocessing involves removing noise inside the signal acquisition from original signal for classification purposes. The db4 was chosen because it was deemed more appropriate for EEG data analysis. The signal's regularity is very important in selecting a wavelet. If the haar wavelet had been chosen, the discontinuity would not have been detected or might be insufficient. However, with db4, the discontinuity can be detected. Signals with regularity have nice features and give smoothness to the reconstructed signals. Also, it helps in properly representing the lower order wavelets of the family as EEG spikes. 20,000 sample data was extracted from original signal with eight levels of decomposition and detailed coefficients were used to analyze the ERPs at P 300 .

\section{RESULTS}

Figure 5 shows the information from sub-band 5 to 8 (gamma, beta, alpha, theta) calculated for further analysis. The information was extracted during the assessment of the study phase. In order to explore the relation between working memory and visual stimulation affects, the averaging of grand mean ERPs value for all 38 healthy young volunteers at the channel $\mathrm{Fz}$ were selected. Since the alpha wave was of major concern, only signals at a decomposition level of 5 (D5) were extracted into the ERPs' signal analysis.

One trial

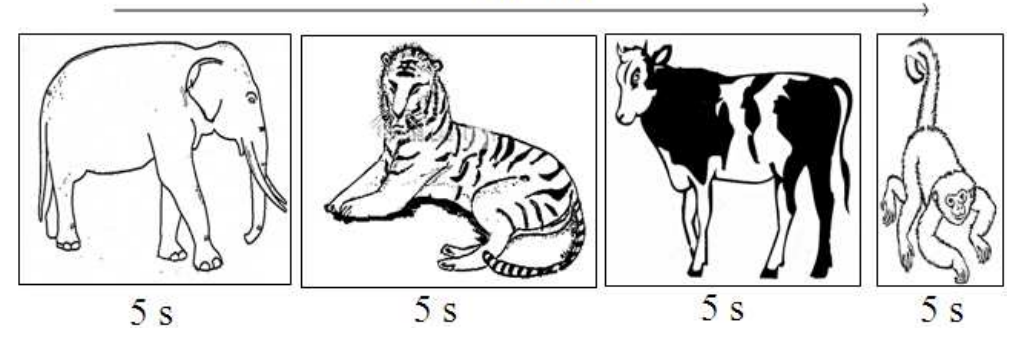

Fig. 2. Study phase 
SitiZubaidahMohd Tumari et al. / Journal of Computer Science 10 (2): 264-271, 2014

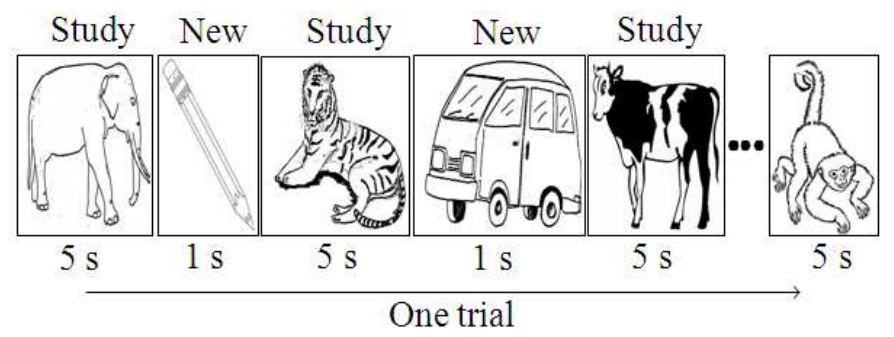

Fig. 3. Working memory test phase

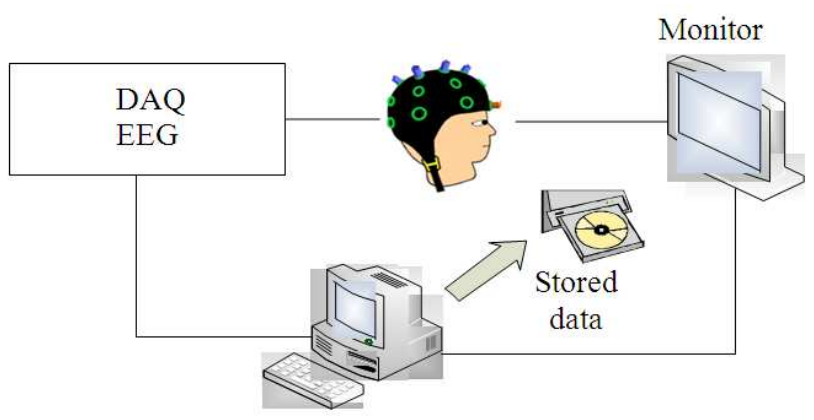

Fig. 4. Experimental setup

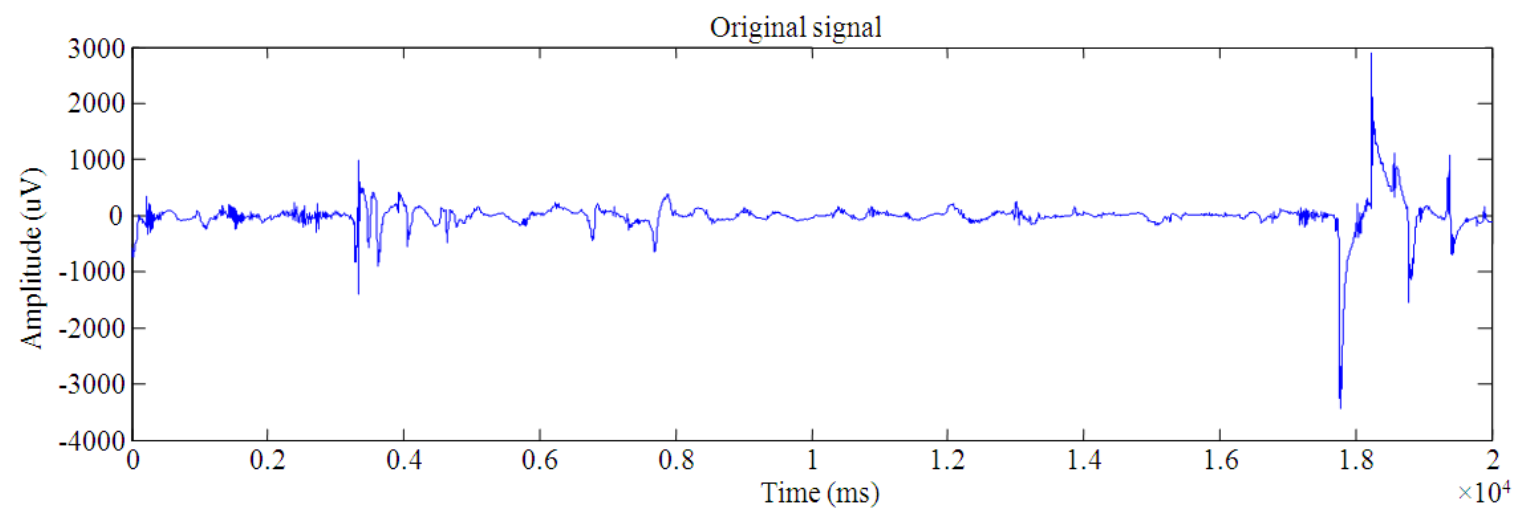

(a)
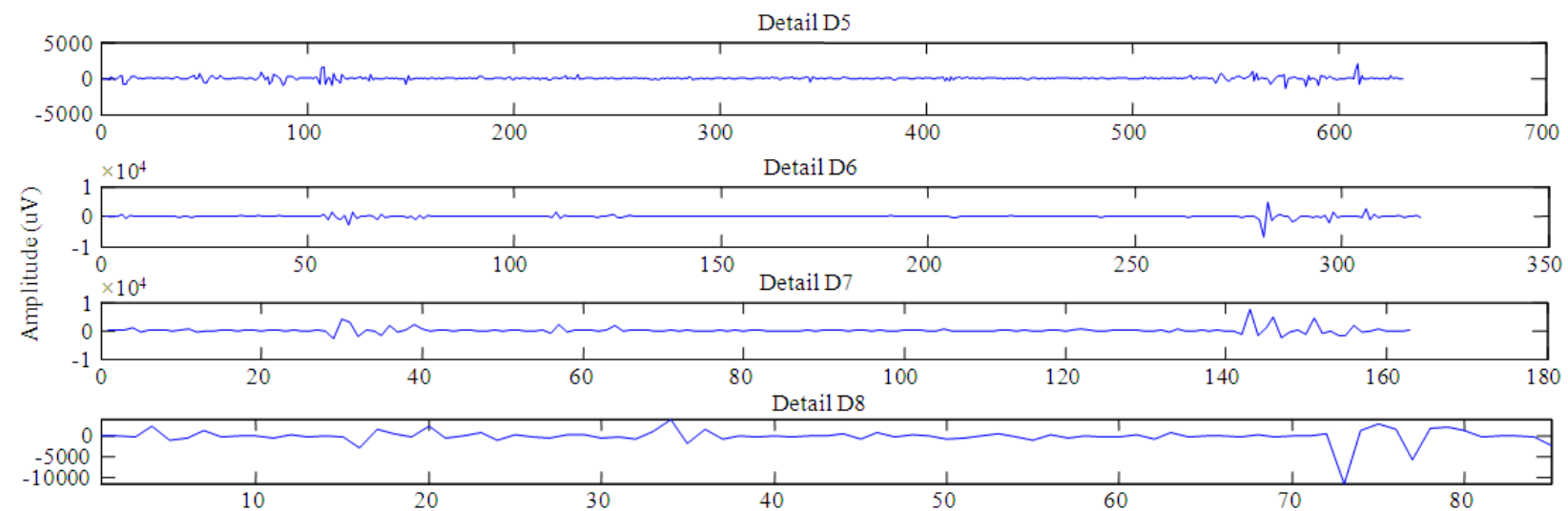

(b) 
SitiZubaidahMohd Tumari et al. / Journal of Computer Science 10 (2): 264-271, 2014

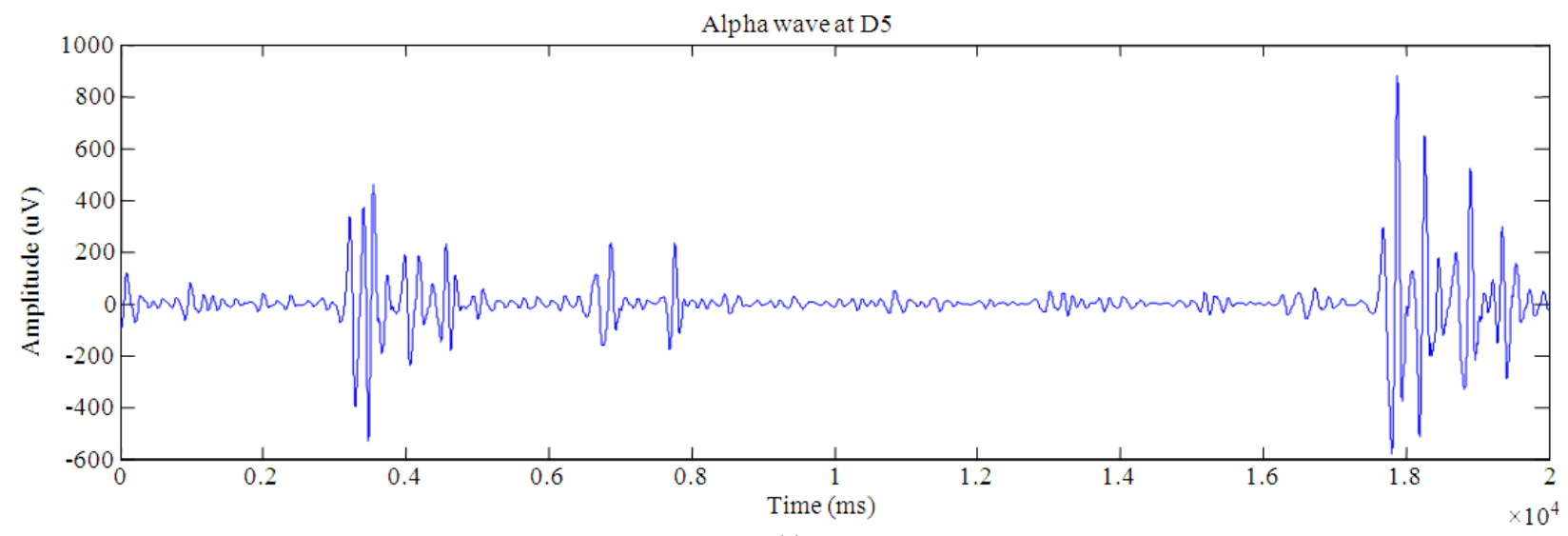

(c)

Fig. 5. EEG extraction using DWT at channel Fz (a) Original signal (b) Detail of coefficients of sub-band 5 to 8 (c) Alpha wave at D5

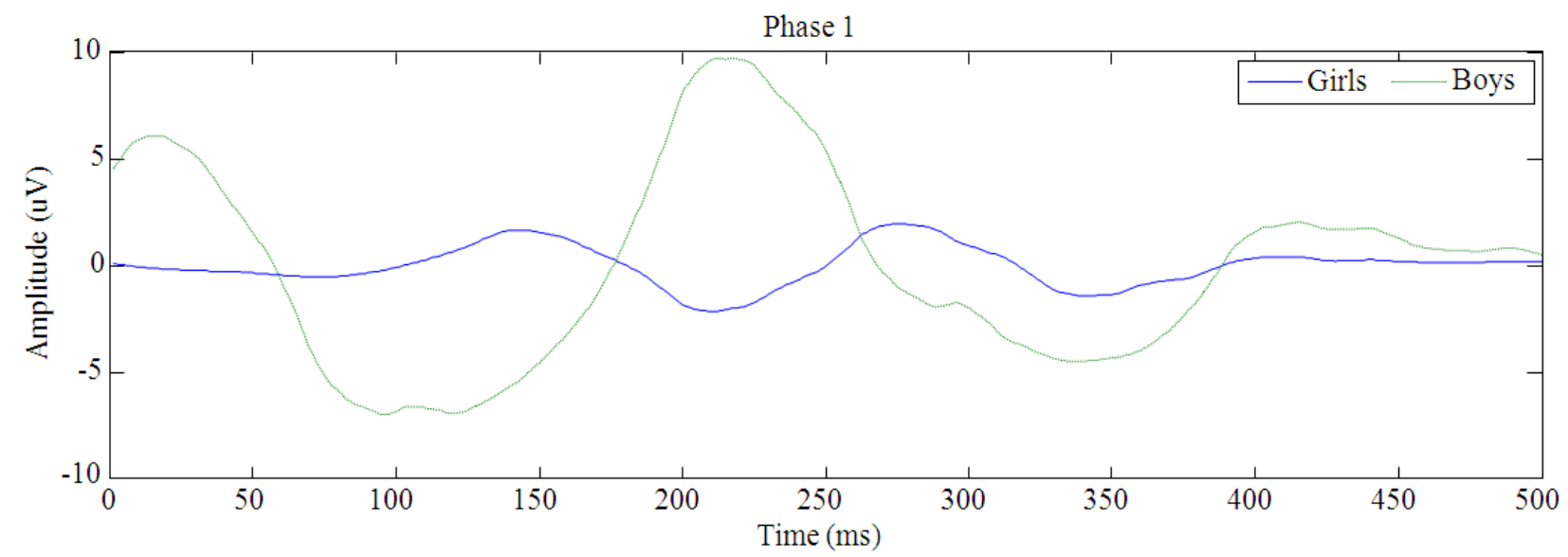

(a)

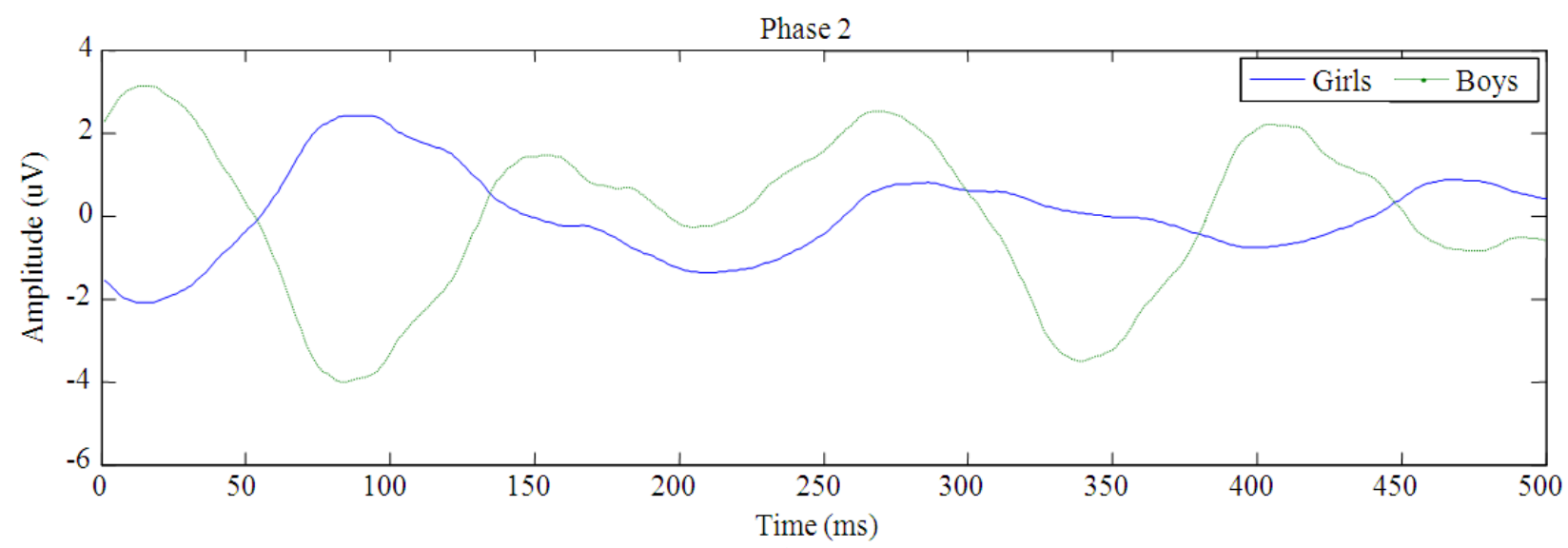

(b)

Fig. 6. Grand average means ERPs at point Fz in a time window from 0 to $500 \mathrm{~ms}$ for alpha wave (a) Phase 1 (b) Phase 2 
At P300, the grand mean ERPs that illustrate the amplitude elicited by visual stimuli for girls was greater than that for boys. Figure 6(a) shows that the girls have higher amplitude at phase $1(0.9145 \mu \mathrm{V})$, but for boys, it was only $-1.999 \mu \mathrm{V}$; the difference about $1.0845 \mu \mathrm{V}$. The amplitude of grand mean ERPs waveform was found to be significantly different by $300 \mathrm{~ms}$ to $450 \mathrm{~ms}$ after the indication response. Figure $\mathbf{6}(\mathbf{b})$ shows that the amplitudes are the same between boys and girls, but the latency for boys delayed when the visual responsiveness became more difficult. The performance on cognitive tasks showed improvement with age, but there was no difference in the magnitude of the old and new ERPs among the 10 to 12 age groups. This also showed that the alpha wave had increased with amplitude variability in the visual stimuli responsiveness.

\section{DISCUSSION}

The study of Cycowicz and Friedman (1999) recorded the ERPs while the subjects were engaged in direct and indirect memory tasks to determine. The participants were between 22 and 28 years old. The task is not suitable for children because their stimulus assessment had more than 128 pictures. Also, due to a lack of equipment and difficulty in locating the frontal lobe area, similar experiments could not be carried out on children at that time to determine that whether they have a poor working memory or not. Cycowicz (2000) came out with a new research that could address children between 9 and 10 years old. During the item recognition test, the time line of waveform was set at every $0.35 \mathrm{sec}$. In that study, the participants achieved a grand mean ERPs of 0.73 and this was less than the mean of young adults (above 12 years old) marked at 0.84 for new pictures tasks. Meanwhile, for old pictures tasks, the children's mean was 0.58 and that of young adults was 0.76 .

A comparison between these previous studies and this study is shown in Table 3. The obvious dissimilarity in that the 12-year-old children had reached a grand mean for ERPs of about 0.21 more than the 10 and 11 year-old children who had reached negative values at P300 during Phase 1. Then, during Phase 2, the 12-yearold children's mean was only 0.02 away from the zero value. The only similarity among all studies is that the results have shown the different brain activity patterns.

Table 3. Grand Mean ERPs for different age at point Fz of the proposed study

\begin{tabular}{llll}
\hline & Age (Years old) & \\
& 10 & 11 & 12 \\
\hline Phase $1(\mu \mathrm{V})$ & 1.79 & 0.05 & 0.21 \\
Phase $2(\mu \mathrm{V})$ & 0.79 & 0.96 & 0.02 \\
\hline
\end{tabular}

Harker and Connolly (2007) involved participants aged between 23 and 24 years old for their studies and the results for ERPs showed a contribution linked to both functional and neurophysiological profiles. Regarding on previous research, this study more focus on the subject from 6 to 12 years old. The visual stimuli assessments were given on the screen rather than using display cards because want to avoid the parallax error of time. The advantages at the end of this study are can differentiate working memory integration with new model of assessments using t-test analysis and can determine the indicator levels of working memory between different ages. To summarize, the major dissimilarity of all studies is in the stimulation material and the way the experiment is conducted. Different subjects give different results, but the grand means for ERPs at P300 are rather close to each other.

\section{CONCLUSION}

Alpha wave signal represents light relaxation, positive thing and fast thinking. The unwanted signal called artifact has to be removed from the raw EEG signal before preprocessing. Higher alpha value leads to higher relaxation. In this study, the Alpha value at Phase 2 had slight delay on latency when the children were exposed to new pictures. Thus, to improve their performance on visual stimuli, they have to be given more time to practice. Training the brain is more important so that the memory can function at relaxed a pace. In conclusion, wavelet functional including localization of the signal in both time and frequency allows ERPs waveform recording. Our future study is to classify the performance of working memory that related with visual stimulation assessments according to the ERPs parameter.

\section{ACKNOWLEDGEMENT}

The researchers would like to express thank all teachers, parents and students whose involve in this study for a useful helpful and encouragement during collect the data. Our appreciation also goes to the Malaysia Ministry of Education, Johor Education Department, Zamalah Scholarship and Universiti Teknologi Malaysia for giving permission, facilities and funding this project.

\section{REFERENCES}

Cycowicz, Y.M. and D. Friedman, 1999. ERP Recordings during a picture fragment completion task: Effects of memory instructions. Brain Res. Cognitive Brain Res., 8: 271-288. DOI: 10.1016/S09266410(99)00031-2 
Cycowicz, Y.M., 2000. Memory development and eventrelated brain potential in children. Biol. Psychol., 54: 145-174. DOI: 10.1016/S0301-0511(00)00055-7.

Donohue, S.E., L.G. Appelbaum, C.J. Park, K.C. Roberts and M.G. Woldorff, 2013. Cross-modal stimulus conflict: The behavioral effects of stimulus input timing in a visual-auditory stroop task. PLoS ONE, 8: e62802-e62802. DOI: 10.1371/journal.pone.0062802

Harker, K.T. and J.F. Connolly, 2007. Assessment of visual working memory using event-related potentials. Clin. Neurophysiol., 118: 2479-2488. DOI: 10.1016/j.clinph.2007.07.026

Krauzlis, R.J., L.P. Lovejoy and A. Zenon, 2013. Superior colliculus and visual spatial attention. Ann. Rev. Neurosci., 36: 165-182. DOI: 10.1146/annurev-neuro-062012-170249

Nenert, R., S. Viswanathan, D.M. Dubuc and K.M. Visscher, 2012. Modulations of ongoing alpha oscillations predict successful short-term visual memory encoding. Front. Hum. Neurosci., 6: 127. DOI: $10.3389 /$ fnhum.2012.00127.

Oliveira, M.O. and A.S. Bretas, 2009. Application of discrete wavelet transform for differential protection of power transformers. Proceeding of the IEEE Burcharest Power Tech Conference, Jun. 28, IEEE Xplore Press, Romania, pp: 1-8. DOI: 10.1109/PTC.2009.5282195

Olkkonen, H., 2011. Discrete Wavelet Transforms: Algorithm and Applications. 1st Edn., In Tech., pp: 308.

Potagas, C., D. Kasselimis and I. Evdokimidis, 2011. Short-term and working memory impairments in aphasia. Neuropsychologia, 49: 2874-2878. DOI: 10.1016/j.neuropsychologia.2011.06.013
Pritzer, B. and F. Blankenburg, 2011. Stimulusdependent EEG activity reflects internal updating of tactile working memory in humans. Proc. Nat. Acad. Sci. USA., 108: 8444-8449. DOI: 10.1073/pnas. 1104189108

Saavedra, C. and L. Bougrain, 2010. Wavelet denoising for P300 single-trial detection. Proceeding of the5th French Conference on Computational Neuroscience, Dec. 1-21, France, pp: 227-231.

Sanei, A. and J.A. Chambers, 2007. EEG Signal Processing. 1st Edn., John Wiley and Sons, ISBN10: 0470025816, pp: 312.

Sudirman, R., A.C. Koh, N.M. Safri, W.B. Daud and N.H. Mahmood, 2010. EEG different frequency sound response identification using neural network and fuzzy techniques. Proceeding of the 6th International Colloquium on Signal Processing and its Applications, May 21-23, IEEE Xplore Press, Malaysia, pp: 1-6. DOI: $10.1109 /$ CSPA.2010.5545237

Tumari, S.Z.M., R. Sudirman and A.H. Ahmad, 2012. Identification of working memory impairments in normal children using wavelet approach. Proceeding of the IEEE Symposium on Industrial Electronics and Applications, Sept. 23-26, IEEE Xplore Press, Indonesia, $\quad$ pp: 326-330. DOI: 10.1109/ISIEA.2012.6496653

Tumari, S.Z.M., R. Sudirman and A.H. Ahmad, 2013. Selection of a suitable wavelet for cognitive memory using electroencephalograph signal. Engineering, 5: 15-19. DOI: 10.4236/eng.2013.55B004

Yang, W., Q. Li, T. Ochi, J. Yang and Y. Gao, 2013. Effects of auditory stimuli in the horizontal plane on audiovisual integration: An event-related potential study. PLoS ONE, 8: e66402-e66402. DOI: 10.1371/journal.pone.0066402 